SEROQUEL * (quetiapine fumarate) Tablets

\section{BRIEF SUMMARY}

TI FOA FULL PRESCRIBING INFORMATION

INDICATIONS AND USAGE

SEROOLEL is indicated for the management of the manifestations of pSychotic disorders.
The antipsychotic efficacy of SEROOUEL was established in short-term CONTAAINDICATIONS

SEROQUEL is contraindicated in individuals with a known hyper WARNINGS

Neuroleptic Malignant Syndrome (NMS): A potentially fatal symptom Neuroleptic Malignant Syndrome (NMS): A potentially latal Symptom
complex sometimes referred to as Neuroleptic Malignant Syndrome (NMS) has been reported in association with administration of antipsy-
chotic drugs. Two possible cases of NMS [2/2387 $(0.1 \%)]$ have been reported in clinical traals with SEROQUEL. Clinical manitestations of NMS are hyperpyrexia, muscie rigidity, altered mental status, and evidence of
autonomic instability (irregular pulse or blood pressure, tachycardia, vated creatinine phosphokinase, myog obinuria (rhabdomyolysis) and acute renal tailure

acte a patient requires antipsychotic drug treatment after recovery from
MMS, the potential reintroduction of drug therapy should be carefully conMMS, the potential reintroduction of drug therapy should be carefully con-
sidered. The patient should be carefully monitored since recurrences of NMS have beent reported.

Tardive Dyskinesia: A syndrome of potentially irreversible, involuntary,
dyckinetic drugs. Althoush the prevalence of the syndrome appears to be highest among the elderly, especially elderly women, it is impossible to rely upon
prevalence estimales to predict, at the inception of antipsychotic treatment. which patients are ikkely to develop the syndrome. Whether antipsychotic If signs and symptoms of tardive dyskinesia appear in a patient on patients may require treatment with SEROQUEL despite the presence of the Syndrome.
PRECAUTIONS: Genera

Orthostatic Hypotension: SEROOUEL may induce orthostatic hypotension associated with dizziness, tachycardia and, in some patients, syncope,
especially durng the initial dose-titration period, probably refiecting its $a_{1}$. especially during the initial dose-titration period, probably refiecting its $\alpha_{1}$.
adrenergic antagonist properties. Syncope was reported in $1 \%(22 / 2162)$ of adrenergic antagonist properties. Syncope was reported in $1 \%$ citients hypotension and syncope may be minimized by limiting the initial dose to $25 \mathrm{mg}$ bid. II hypotension occurs during titration to the target dose, a return to the previous dose in the titration schedule is appropriate.
SEROQUEL should be used with particular caution in patients with known cardiovascular disease (history of myocardial infarction or ischemic hear or conditions which would predispose patients to hypotension (dehydration hypovolemia and treatment with antihypertensive medications).
Cataracts: The development of cataracts was observed in associa-
tion with quetiapine treatment in chronic dog studies. Lens changes tion with quetiapine treatment in chronic dog studies. Lens changes have also been observed in patients during long-term SEROQUEL treal-
ment, but a causal relationship to SEROQUUEL use has not been establisher. Nevertheless, the possibility of lenticular changes cannot be excluded at this time. Therefore, examination of the lens by methods appropriately sensitive methods, is recommended at initiation of treat-
ment or shortly thereafter, and at 6 month intervals during chronic treatment.

Seizures: As with other antipsychotics SEROQUEL should be used cautiously in patients with a history of seizures or with conditions that potenthat lower the seizure threshold may be more prevalent in a population of

Hypothyroidlsm: Clinical trials with SEROOUEL demonstrated a doseto four iweeks of treatment and maintained without adaptation or wo gression during more chronic therapy. Generally, these changes were of no clinical significarice and TSH was unchlanged in most patients, and lev-
els of TBG were unchanged. In nearly ail cases, cessation of SEFOOUEL els of TBG were unchanged. In nearly all cases, cessation of SEROQUEE
treatment was asscciated with a reversal of the elfects on total and free T4 treatment was asscciated with a reversal of the encut $0.4 \%(10 / 2386)$ of of
irrespective of the duration of treatment. About 0.4 .
SEROOUEL patients did experience TSH increases. Six of the patients with TSH increases needed replacement thyroid treatment. with TSH increases needed replacement thyroid treatment.
Cholesterol and Iriglyceride Elevations: In a pool of 3- to 6-week
placebo-controlled trials SEROOUEplacebo-controlled trials SEROOUEL-treated patients had increases from compared to slight decreases tor placebo patients. These changes were
only weakly related to the increases in weight observed in SEROQUEL.

Hyperprolactinemia: Although an elevation of prolactin levels was not
demonstrated in clinical trials with SEROOUEL, increased prolactin levels were observed in rat studies with this compound, and were associated with an increase in maminary gland neoplasta in rats (see
Carcinogenesis). Tissue culture experiments indicate that approximately Carcinogenesis). Tissue culture experiments indicate that approximately
one--third of human breasi cancers are proiactin dependent in vitro, a tacplated in a patient with previously detected breast cancer. Although disturbances such as galactornea, amenor hea, gynecomastia, and impotence have been reported with prolactin-elevating compounds, the clinical significance of elevated serum prolactin levels is unknown for most
patients Neither clinical studies nor epiderniologic studies conducted to patients. Neither clinical studies nor epiderniologic studies conducted to
date have shown an association between chronic administration of this class of drugs and tumorigenesis in humans: the
considered too limited to be conclusive at this time. Transaminase Elevations: Asymptomatic, transient and reversible
elevations in serum transaminases (primarily ALT) have been reported.
The proportions of patients with transaminase elevations of $>3$ times the The proportions of patients with transaminase elevations of $>3$ times the
upper limits of the normal reference range in a pool of 3 - to 6 -week placebo-Controlled trials were approximately $6 \%$ for SEROQUEL Compared to
$1 \%$ for placebo. These hepatic enzyme elevations usually occurred within $1 \%$ for placebo. These hepatic enzyme elevations usually occurred within
the first 3 weeks of drug treatment and promptly returned to pre-study levels with ongoing treatment with SEROOLE

Potential for Cognitive and Motor Impairment: Somnolence was a Commonly reported adverse event reported in patients treated with
SEROQUEL especially during the $3-5$ day period of initial dose-litration In the 3- to 6-week placebo-controlled trials, somnolence was reported in $18 \%$ of oatients on SEROQUEL Compared to $11 \%$ of placebo patients
Since SEROQUEL has the potential to impair judgment, thinking, or moto skills, patients should be cautioned about performing activities requiring
mental alertness. such as operating a motor vehicle (including automobiles mental alertness, such as operating a motor vehicle (including automobiles
or operating hazardous machinery until they are reasonably certain tha SEROAUEL therapy does not aftect them adversely.
Priapism: One case of priapism in a patient receiving SEROQUEL has been repotted prior to market introduction.
Body Temperature Regulation: Although not reported with SEROOUEL Body Temperature Regulation: Although not reported with SEROOUEL,
disruption of the body's ability to reduce core body temperature has been Dysphagia: Esophageal dysnotility and assiration have been associated with antipsychotic drug use. Aspiration pneumonia is a common cause of advanced Alzheimer's dernentia. SEROQUEL and other antipsychotic drug should be used cautiously in patients at risk for aspiration pneumonia.
Suicide: The possibility of a suicide attempt is inherent in schizophre-

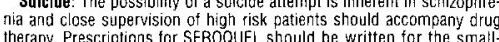
est cuantity of tablets consistent with good patient management in order to reduce the risk of overdose.
ond
Use in Patients with Concomitant Illness: Clinical experience with SEROOUEL in patients with certain concomitant systemic illnesses is limited. SEROOUEL has not been evaluated or used to any appreciable extent in
patients with a recent history of myocardial infarction or unstable heart patents with a recent history of myocardia infarction or unstable heaf
disease. Patients with these diagnoses were excluded from premarketing clinical studies. Because of the risk of orthostatic hypotension with SEROQUEL. caution should be observed in cardiac patients (see Orthostatic Hypotension).
Intormation for Patient

Physicians are advised to discuss the following issues with patients for whom they prescribe SEROQUEL.
Orthostatic Hypotension: Patients should be advised of the risk of orthoOrhostatic Hypotension: Patients should be acvised of the risk of orhe-
static hypotension, essecially during the 3-5 day period of initial dose titration, and also at times of ere-initiating treatment or increases in dose.
Interference with Cognitive and Motar Performance: Since somnolence
was a commonly yeported adverse event associated with SEROQUEL treatwas a commonly reported adverse event associated with SEROQUEL treating the 3-5 day period of initial dose tifration. Patients shouid be cautioned
about performing any activity requiring mental alerthess, such as operating about performing any activity requiring mental alerness, such as operating
a motor vehicle (including automobiles) or operating hazardous machinery, thern adversely.
Pregnancy: Fatients should be advised to notify their physician it they Pregnancy: Patients should be advised to notify their physician if they
become pregnant or intend to become pregnant during therapy.
Nursing: Patients should be advised not to breast feed if they are taking Concomitant Medication: As with other medications, patients should be Concomitant Medication: As with other medications, patients sholid be
advised to notify their physicians if they are taking, or pian to take, any prescription or over-the-counter drugs. Alcohol: Patients shculd be ad

beverages while taking SEROQUEL.
Heat Exposure and Dehydration: Patients snould be advised regarding appropriate care in avoiding overheating and dehydration.

aboratory Tests

No specific laboratory tests are recommended. The risks of using SEROCUEL in combination with other drugs have not
been extensively evaluated in systematic studies. Given the primary CNS effects of SEROQUEL, caution should be USed when it is taken in combination with other centrally acting drugs. SEROOUEL potentiated the cogselected psychotic disorders, and alconolic beverages should be avoidBecause of its potential for inducing hypotension, SEROQUEL may enhance the effects of cerlain antihypertensive agents.
SEROQUEL may antagonize the effects of levodopa and dopamine The Eliect of Other Drugs on SEROQUEL

Phenytoin: Coadministration of quetiapine (250 $\mathrm{mg}$ tid) and phenytoin Increased doses of SEROQUEL may be required to maintain control of psychotic symptoms in patients receiving quetiapine and phenytoin, or other hepatic enzyme inducers (e. g., carbamazepine, barbiturates.
rifampin, glucocorticoids). Caution should be taken if phenytoin is withdrawn and replaced with a nort-inducer (e.g. valoroate).
Thioridazine: Thioridazine (200 mg bid) increased the oral clearance of Thioridazine: Thioridazine $(200 \mathrm{mg}$ bid) increased the oral clearance of
quetiapine (300 mg bid) by $65 \%$. quetiapine ( $300 \mathrm{mg}$ bid

P450 $3 \mathrm{~A}$ Inhibitors: $\mathrm{Coadministration} \mathrm{of} \mathrm{ketoconazole} 200 \mathrm{mg}$ once

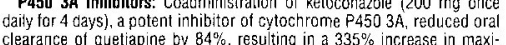
rrmum plasma concentration of quetiapine. Caution is indicated when
SEROOUEL is administered with ketoconazole and other inhibitors of cytochrome $\mathrm{P} 4503 \mathrm{~A}$ (e.g. Itraconzaole, fluconazole, and erythromycini.
Fluoxetine, Imipramine, Haloperidol, and Risperidone

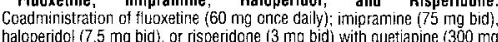
haloperidol ( $7.5 \mathrm{mg}$ bid), or risperidone ( $3 \mathrm{mg}$ bid) with quetiapine
bid) did not alter the steady-state pharmacokinetics of quetiapine. ffect of Quetiapine on Other Drugs

Lerazepam: The mean oral clearance of lorazepam ( $2 \mathrm{mg}$. single dose)
was reduced by $20 \%$ in the presence of quetiapine adrministered as $250 \mathrm{mg}$ was reduced by $20 \%$ in the presence of quetiapine administered as $250 \mathrm{~m}$
tio dosing. Lithium: Concomitant administration of quetiapine $(250 \mathrm{mg}$ tid) with lithium had no effect on any of the steady state pharrmacokinetic parame-
ters of lithium.
Antipyrine Sudy results indicate that quetiagine does not significantly Antipyrine: Study results indicate that quetiapine does not significantly
induce hepatic enzymes responsible for cytoctrorre P450 mediate induce hepatic enzymes respons blabolism of antipyrine.

Carcinogenesis, Mutagenesis, Impairment of Fertility
Carcinogenesis: Carcinogenicity studies were conducted in C57BL mice and Wistar rats.

The relevance of the increases in thyroid follicular cell adenomas to human risk, through whatever mechanism, is unknown.
The relevance of this increased incidence of protactin-mediated mammay gland tumors in rats to human risk is unknown (see Hyperprolactinemia Mecallons, Gene

Mutagenesis: Quetiapine did produce a reproducible increase in mut tiont in one Salmone No zophimurium taster strain in the presence of
metabolic activation. Ve vidence of clastogenic potential was obtained in
an in vitro chromosomal aberration assay in cultured human lymphocytes

Impairment of Fertility: Drugg-re inted effects included decreases in
matings and in matings resulting in pregnancy, and an increase in the matings and in matings resulting in $p$
interval to mate.

Pregnancy: Pregnancy Category $C$

trolled studies in pregnant women Jisk during pregnancy only if the potential benLabor and Delivery: The effect of SEROQUEL on labor and delivery in humans is unknown:
Nursing Mothers: SEROCUEL was excreted in milk of treated animals during lactation. It is not known if SEROQUEL is excreted in human milk. Pediatric Use: The satety and effectiveness of SEROQUEL in pediatric patients have not been established.

Geriatric Use: Of the apporoximately 2400 patients in clinical studies
with SEROQUEL, $8 \%$ (190) were 65 years of age or over In general there with SEROQUEL, $8 \%$, 190 ) were 65 years of age or over, In general, there ly compared to younger adults. Nevertheless, the presence of factor in should lead to consideration of a lower starting dose. slower titration. and careful monitoring during the initial dosing period in the elderly. The elderly patients when co

Advers Events Occurring at an Incidence of $1 \%$ or More Among SEROQUEEL Treated Patients in Shart-Ferm, Placebo-Controlled Trials:
The most commonly observed adverse events associated with the use of
SEROOUEL (incidence of $5 \%$ or greater) and observed at a rate on SEROOUEL at least twice that of olacebo were dizziness $(10 \%)$. postual hypotension $(7 \%)$, dry mouth $(7 \%)$, and dyspepsia $(6 \%)$. incidence rate of $\%$ or more, and were at least as frequent among
SEROOUEL treated patients, treated at doses of 75 mg/day or greater than SEROOUEL lreated patients, treated at doses of $75 \mathrm{mg} /$ day or greater than mong placebo treated patients in 3- to 6-week placebo-controlled triais.
Body as a Whole: Headache, Asthenia, Abdominal pain, Back pain,
Fever, Nervous System: Somnolence, Dizziness; Digestlue System Fever, Nervous System; Somnolence, Dizziness; Digestlve System: hypoternion, Tachycardia: Mietabolic and Mutritional Disorders: Weight
gain; Skin and Appendages: Rash; Respitatory System: Rninitis:
SEROOUEL (quetiapine fumarate) Tablets

Special Senses: Ear pain

Events for which the SEROQUEL incidence was equal to or less tha placebo are nol listed, but included the following: pain, infection, ches:
pain, hostility, accidental injury, hypertension, nypotension, nausea vomiting, diarrhea, myalgia, agitation, insomnia, anxiety, nervousness. skin, amblyopia and urinary tract infection.
Dose Dependency of Adverse Events in Short-Term, Placebo Controlled Trias

Dose-related Adverse Events: Spontaneously elicited adverse even data from a study comparing five fixed doses of SEROQUEL $(75 \mathrm{mg}$ dose-relatedness of adverse events. Logistic regression analyses revealed pepsia, abdominal pain, and weight gain. Extrapyramidal Symploms: Data from one 6-week clinical trial comparing
five fixed doses of SEROQUEL $(75,150,300,600,750 \mathrm{mg} / \mathrm{day})$ provided evidence for the lack of treatment-emergent extrapyramidal symptoms
(EPS) and dose-relatedness for EPS associated with SEROQUEL treatment. Three methods were used to measure EPS (1) Simpson-Angus total score (mean change trom baseline) which evaluates parkinsonis and akathisia. (2) incidence of spontaneous complaints of EPS (akathisia, akinesia. cogwtheyl rigidity, extrapyramidal syndrome, hyper-
tonia, hypokinesia, neck rigidity, and tremor), and (3) use of anticholiner-

gic medications to treat emergent EPS.
Vital Sign Changes: SEROCUEL is associated with orthostatic hypoten(See PRECALTIONS)

Weight Gain: The proportions of patients meeting a weight gain criterion of $\geq 7 \%$ of body weight were compared in a pool of four 3- to 6 -week placedence of weight gain for SEROQUEL (23\%) compared to placebo $(6 \%)$. Laboratory Changes: An assessment of the premarketing experience
for SEROQUEL suggested that it is associated with asymptomatic increases in SGPT and increases in both total cholesterol and triglyc-
erides (see PRECAUTIONS. An assessment of hematological parameters in short-term. placebocontrolled trials revealed no clinically important differences between EROQUEL and placebo.

ECG Changes: Between group comparisons for pooled placebo-controlled
trals revealed no statistically significant SEROQUEL $L$ placebo ditterences in the proportions of patients experiencing potentially important changes in EG parameters, including QT, QTC, and PQ intervals. However, the propor3- to 6 -week dence for SEROQUEL compared to $0.6 \%$ (1/156) incidelice for placeso SERCOUEL use was associated with a mean inc isase in heart rate, assessed by ECG, of 7 beats per minute compared to a mean increase of 1 beat per minute among placebo patients. This slight tendency to tachycardia may be
related to SEROQUELL's potential for inducing orthostatic changes (see PAE-

Other Adverse Events Observed During the Pre-Marketing Evaluation Following is a list of COSTART terms that reflect treatment-energent
dverse events as defined in the introduction to the ADVERSE REACTIONS section reported by patients treated with SEROQUEL at multiple doses $\geq 75 \mathrm{mg} / \mathrm{day}$ during any phase of a trial within the premarketing data.
base of approximately 2200 patients. All reported events are included except those already listed in Table 1 or elsewhere in labeing, those events
for which a drug cause was remote, and those event terms which were so general as to be uninformative. It is importanl to emphasize that, although ot necessarily caused by i

Events are further categorized by body system and listef in order of decreasing frequency.
Nervous System: Frequent: hypertonia, dysarthria: Infrequent: abnor ina dreams, dyskinesia, thinking abnorma!, tardive dyskinesia, vert go, Involuns, myperkinesiav, libido increased ${ }^{*}$. amnesia, psychosis, hal ucinaparanoid reaction, abnormal gait, myoclonus, delusions. manic reaction, apathy, ataxia, depersonalization, stupor, bruxism, catatonic reaction, hemiplegia: Rare: aphasia, buccoglossal syndrome, chore aathetosis,
delirium, emotional lability, euphoria, libido decreased", neuralgia, stut-

Body as a Whole: Frequent: f| s syndrome: Infrequent: neck pain, pelvic pain $^{*}$. suicide attempt. malaise, photosensitivity reaction, chills, face Digestive System: Frequent: anorexia; infrequent: increased salivation increased appetite, gamma glutamyl transpeptidase increased, gingivitis,
dysphaoia, flatulence, gastroenteritis, gastritis, hemorrhoids, stomatitis, dysphagia, flatulence, gastroenteritis, gastritis, hemorrhoids, stomatit is,
thirst, tooth caries. fectai incontinence, gastroesophageal reflux. gurn hemorrhage, mouth ulceration. rectal hemorhage, tongue edema Cardioyascular System: Frequent: obstruction. melena, pancreatitis latation, of interval prolonged, migraine, bradycardia, cerebrał: ischemia, irregular pulse, T wave abnormality, bundle branch block, cerebrovascu-
lar accident, deep thrombonhlebitis. T wave inversion; Bare: angina peelar accident, deep thrombophtebitis, T wave inversion; Bare: angina pee-
toris, atrial fibrillation, AV block first degree, congestive heart tail lure. toris, atrial fibrillation, AV block first degree, congestive heart tailure,
ST elevated, thrombophlebitis. T wave flattening, ST abnormality, Respiratory System: Frequent: pharyngitis, rhinitis, cough increased
dyspnea; Infrequent: pneumonilia, epistaxis, asthma: Rare: hiccup, hyperventilation.

Metabolic and Nutritional System: Frequent: peripheral edena Infrequent: weight: loss, alkaline phosphatase increased, hyperlipemia,
alcohol intolerance, dehydration. hyperglycemia, creatifine increased hypoglycemia; Rare: glycosuria, gout, hand edema, hypokalemia, wate

Skin and Appendages System: Frequent: sweating: Infrequent: pruti tis, acne, eczema, contact dermatitis, maculopapular rash, seborrhea, skin

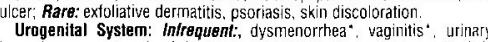

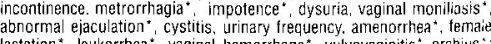
Rare: gynecomastia*, nocturia, polyuria, acute kidney failute.
Special Senses: Intrequent: conjunctivitis, abnurrmal vision, dry
eyes, tinnitus, taste perversion, blepharitis, eye pann, Rare: abnormality Musculoskeletal System: imtrequent; pathological fracture. myasthe nia, twitching, arthralgia, arthritis, leg cranips, bone pain.
Hemic and Lymphatic System: frequent: leukopenia; infrequent. Hemlt and Lymphatic System: Frequent: leukopenta, Intequemia
leukocytosis, anemia, ecchymosis. eosinophilia, nypochromic anemia lymphade nopathy, cyanosis; Rare: hemolysis, thrombocytopenia.
Endocrine System: Infrequent: hypothyroidism, diabetes mellitus Rare: hyperthyroidism.

Post Marketing Experlence: Adverse events reported since market Post Marketing Experlenes: Adverse events reported since market
introduction which were temporally related to SEROQUEL therapy include the iollowing: rarely leukopenia/neutropenia. If a patient develops a low white cell colunt consider discontinuation of therapy. Possible
risk factors for leukopenia/neutropenia include pre-existing low white cell
count and history of drug ind uced leukopeniaineutropenia.

\section{DRUG ABUSE AND DEPENDENCE}

All trademarks are the property of the Astrazeneca Group.

Rer L 0990

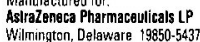




\section{In first-line antipsychotic therapy...}

\section{Imagine life with less EPS}

\section{Outstanding Efficacy}

- The strength to control positive, negative, and overall symptoms of psychosis ${ }^{1,2}$

- Improves depressive symptoms associated with psychosis ${ }^{3 *}$

\section{Less EPS}

- No different from placebo across the entire dose range ${ }^{1,4}$

-Adjust dose without increasing risk of EPS

-Minimal need for anticholinergic medications ${ }^{4,5}$

\section{Minimal Weight Gain ${ }^{4,5}$}

\section{Maintenance Dosing \\ -Initial dose range is $300 \mathrm{mg} /$ day to $400 \mathrm{mg} / \mathrm{day}^{4}$ \\ -Further adjustments up to $800 \mathrm{mg} /$ day when needed ${ }^{4}$}

In placebo-controlled trials, the most common adverse events leading to treatment withdrawal were somnolence $(0.8 \%)$ and hypotension $(0.4 \%)$.

Consideration should be given to a slower rate of titration and a lower target dose in the elderly and other special populations.

As. with all antipsychotic medications, prescribing should be consistent with the need to minimize the risk of tardive dyskinesia, seizures, and orthostatic hypotension. ${ }^{4}$

The safety and effectiveness of SEROQUEL in pediatric patients have not been established. ${ }^{4}$

Rlease see brief summary of full prescribing information on the following page.

*Improves depressive symptoms as measured by the Mood Cluster Score of the Brief Psychiatric Rating Scale (BPRS), a clinical assessment tool that measures a combinatjon of 18 individual positive, negative, and general symptom items such as conceptual disorganization, hallucinatory behavior, depressive mood, hostility, suspiciousness, and anxiety.

Referencel: 1. Akvanitis LA, Miller BG, Seroquel Trial 13 Study Group. Multiple fixed doses of "Seroquel" (quetiapine) in patients with acute exacerbation of schizophrenia: a comparison with haloperidol and placebo. Biol Psychiatry. 1997;42:233-246. 2. Small JG, Hirsch SR, Arvanitis LA, Miller BG, Link CGG, Seroquel Study Group. Quetiapine in patients with schizophrenia: a high- and low-dose double-blind comparison with placebo. Arch Gen Psychiatry. 1997;54:549-557. 3. Goldstein JM. Quetiapine fumarate (Seroquel "): a new atypical antipsychotic. Drugs of Today. 1999;35(3): 193-210. 4. SEROQUEL (quetiapine fumarate) Professional Information Bìachure, Zeneca Pharmaceuticals, A Business Unit of Zeneca Inc, Wilmington, Delaware. 5. Data on file, Quetiapine (SEROQUEL) Experience with Safety and Tolerability (QUEST), AstraZeneca Pharmaceuticals LP, Wilmington, Delaware

www.seroquel.com AstraZeneca

AstraZeneca Pharmaceuticals LP
Start with... Switch to...Stay with.

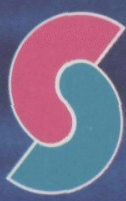

Seroquel quetiapine fumarate 


\section{SUBSCRIPTION INFORMATION}

\section{$\square$ YES! I want to continue my free subscription to CNS SPECTRUMS}

Name:

Address:

E-mail:

Specialty:

Signature:
FAX: 212.328.0600

MAIL:

CNS SPECTRUMS

MedWorks Media

333 Hudson Street, 7th Floor

New York, NY 10013

\section{PAID SUBSCRIPTIONS}

$\square$ Primary PSYCHIATRY

First in Applied Psychiatric Medicine

12 issues per year. One-year subscription rate:

Domestic: \$90; Foreign: \$145; In-training: \$50 (2001)

\section{$\square$ TEN}

\section{The Economics of Neuroscience}

12 issues per year. One-year subscription rate:

Domestic: \$120; Foreign: \$185; In-training: \$75

\section{$\square$ ONCOLOGY SPECTRUMS}

\section{The Journal of Integrated Cancer Medicine}

12 issues per year. One-year subscription rate:

Domestic: \$120; Foreign: \$185; In-training: \$75

\section{Billing}

$\square$ Invoice Me

$\square$ Visa $\square \mathrm{MC} \square \mathrm{AmEx}$

Name:

Card \#:

Exp. Date:

\section{Gontinuing Medical education}

Category 1 credits for psychiatrists, primary care physicians, and neurologists are available each month in Primary Psychiatry and CNS Spectrums, as well as on the MedWorks Media Web site:

\section{www.medworksmedia.com}

To participate, complete and mail the test and registration forms that are included in every issue of Primary Psychiatry and CNS Spectrums, or visit our Web site. 


\section{Introduction}

CNS Spectrums is a peer-reviewed journal that publishes original scientific literature and reviews on a wide variety of neuroscientific topics of interest to the clinician. CNS Spectrums publishes 12 issues in 2001. As the immense prevalence of comorbid diseases among patients seen by psychiatrists and neurologists increases, these physicians will jointly diagnose and treat the neuropsychiatrically ill. Our mission is to provide these physicians with an editorial package that will enhance and increase their understanding of neuropsychiatry; therefore, manuscripts that address crossover issues germane to neurology and psychiatry will be given immediate priority.

\section{Scope of Manuscripts}

CNS Spectrums will consider the following types of articles for publication:

Original Reports: Original reports present methodologically sound original data.

Reviews: Reviews are overview articles that summarize and synthesize the literature on various topics in a scholarly and clinically relevant fashion. Suitable topics include mood disorders, schizophrenia and related disorders, personality disorders, substanceuse disorders, anxiety disorders, neuroscience, psychosocial aspects of psychiatry, child psychiatry, geriatric psychiatry, and other topics of interest to clinicians. nb: Original flowcharts designed to aid the clinician in diagnosis and treatment will be considered for publication in reviews and are encouraged.

Case Reports: Single or multiple case reports will be considered for publication.

Letters to the Editor: Letters will be considered for publication.

\section{Manuscript Submissions}

General information: Four copies of the manuscript should be submitted to Jack M. Gorman, editor (or, in Europe, to Joseph Zohar, international editor), c/o MedWorks Media, 333 Hudson Street, 7th Floor, New York, NY 10013; T: 212.328.0800, F: 212.328.0600. Authors are required to submit their manuscripts on computer disks. If possible, please provide them in MS Word for Windows in either a Macintosh or IBM format. (Saving the file in a lower version, eg, MS Word 3.0, is also encouraged.) Disks should be labeled with the word-processing program, title of paper, and first author's name.

Letters of permission to reproduce previously published material: All material reproduced from previously published copyrighted material must be accompanied by a letter of permission from the copyright holder. All such material should include a full credit line (eg, in the figure or table legend) acknowledging the original source. Any citation of unpublished material or personal communication should also be accompanied by a letter of permission for anyone who is not an author of the paper.

Peer review: Authors should provide five names of particularly qualified potential reviewers with no conflict of interest in reviewing the work. Contact information, including complete address, phone, fax numbers, E-mail address, and affiliations, should be included. The corresponding author will be notified by the editors when a decision regarding acceptance has been made. Accepted manuscripts and letters will be edited for clarity and style.

\section{Manuscript Preparation}

Length: Reviews should not exceed 20 manuscript pages $(10,000$ words). Original reports should not exceed 15-25 manuscript pages (6,250 words, maximum). Letters should not exceed 2-6 manuscript pages (1,500 words, maximum). Single case reports should not exceed 10-15 manuscript pages (3,750 words, maximum) and may be submitted with a photograph, if applicable. Diagnostic/treatment algorithms (see Reviews) should contain an extensive introduction, a flowchart or series of graphs that fill 8-12 journal pages, and a concise summary.

Spacing: One space should be left after commas and periods. Manuscripts should also be double-spaced.

Abstract: Authors should provide a brief abstract.

References: American Medical Association style. See the following examples:

1. Jones J. Necrotizing Candida esophagitis. JAMA. 1980;244:2190-2191.

2. Stryer L. Biochemistry. 2nd ed. San Francisco, Calif: WH Freeman Co; 1980:559-596.

Copyright: Materials are accepted for exclusive publication in CNS Spectrums and become the property of CNS Spectrums. Permission to reproduce material must be obtained from the publisher.

\section{Disclosure of Commercial Interests}

The authors must include a statement about all forms of support, including grant and drug company support. Such information may, at the editor's discretion, be shared with reviewers. If the article is accepted for publication, the editors will consult with the authors as to whether this information should be included in the published paper.

Reprints: Authors of reviews and original materials published in CNS Spectrums may order reprints of their articles directly from the publisher, James La Rossa Jr., MedWorks Media, 333 Hudson Street, 7th Floor, New York, NY 10013; T: 212.328.0800, F: 212.328.0600.

Continuing Medical Education requirements: Authors must submit four multiple-choice questions (two Type $A$ and two Type K) with answers.

\section{Submission Checklist}

1. Original manuscript plus copies

2. Copies of permission letters to reproduce previously published and unpublished material

3. A brief abstract of article.

4. Two multiple-choice questions with answers

5. Disk labeled with the word-processing program, title of paper, and first author's name

6. Names and addresses of five potential reviewers. 


\section{GuIDE to DSM-IV ANd ICD-10 Codes}

Dementia of the Alzheimer Type, With Early Onset With Depressed Mood

DSM-IV

ICD-10

Specify if: With Behavioral Disturbance

290.13

F00.03

Specify if: With Behavioral Disturbance

Delirium Due to: Indicate General Medical Condition

Psychotic Disorder Due to: Indicate General Medical Condition With Delusions

With Hallucinations

Mood Disorder Due to: Indicate General Medical Condition

Anxiety Disorder Due to: Indicate General Medical Condition

Amnestic Disorder Due to: Indicate General Medical Condition

Dementia NOS

Amnestic Disorder NOS

Schizophrenia

Schizophrenia-Disorganized Type

Schizophrenia-Catatonic Type

Schizophrenia-Paranoid Type

Schizophrenia-Residual Type

Schizoaffective Disorder

Schizophrenia-Undifferentiated Type

Major Depressive Disorder

Bipolar I Disorder

Bipolar Disorder NOS

Bipolar II Disorder

Mood Disorder NOS

Psychotic Disorder NOS

Autistic Disorder

Asperger's Disorder

Pervasive Developmental Disorder NOS

Anxiety Disorder NOS

Panic Disorder Without Agoraphobia

Generalized Anxiety Disorder

Dissociative Identity Disorder

Dissociative Disorder NOS

Factitious Disorder NOS

Panic Disorder With Agoraphobia

Agoraphobia Without History of Panic Disorder

Social Phobia

Specific Phobia

Obsessive-Compulsive Disorder

Dysthymic Disorder

Depersonalization Disorder

Body Dysmorphic Disorder

Somatization Disorder

Somatoform Disorder NOS

Cyclothymic Disorder

Alcohol Dependence

Cocaine Dependence

Cannabis Dependence

Amphetamine Dependence

Alcohol Abuse

Cannabis Abuse

Cocaine Abuse

Amphetamine Abuse

Stuttering

Anorexia Nervosa

Tic Disorder NOS

Tourette Disorder

Primary Insomnia

Primary Hypersomnia

Sleepwalking Disorder

Dyssomnia NOS

Nightmare Disorder

Parasomnia NOS

Eating Disorder NOS

Bulimia Nervosa

Feeding Disorders of Infancy or Early Childhood

Communication Disorder NOS

Posttraumatic Stress Disorde

Depressive Disorder NOS

Impulse-Control Disorder NOS

Pathological Gambling

Pyromania

Kleptomania

Trichotillomania

Disruptive Behavior Disorder NOS

Attention-Deficit/Hyperactivity Disorder, Combined Type

Attention-Deficit/Hyperactivity Disorder NOS

Learning Disorder NOS

Developmental Coordination Disorder

Narcolepsy

Sleep Disorder Due to: Indicate General Medical Condition

Delirium NOS

290.2

293.0

293.81

293.82

293.83

293.89

294.0

294.8

294.8

295

295.20

295.30

295.60

295.70

295.9

296

296.80

296.89

296.90

298.9

299.00

299.80

299.80

300.00

300.01

300.02

300.14

300.15

300.19

300.21

300.22

300.23

300.29

300.3

300.4

300.6

300.7

300.81

300.81

301.13

303.90

304.20

304.30

304.40

305.00

305.20

305.60

305.70

307.0

307.1

307.20

307.23

307.42

307.44

307.46

307.47

307.47

307.47

307.50

307.51

307.59

307.9

309.81

311

312.30

312.31

312.33

312.34

312.39

312.9

314.01

314.9

315.9

347

347
780

780.09

F00.13

F05.0

F06.2

F06.O

F06

F06.4
F02.8

$\mathrm{FO} 3$

R41.3

F20

F20.1

F20.2

F20.0

F20.5

F25

F20.3

F32

F30

F39

F31.8

F39

F29

F84

F84.5

F84.9

F41.9

F41

F41.1

F44.81

F44.9

F68.1

F40.01

F40

F40.1

F40.2

F42.8

F34.1

F48.1
F45.2

F45.

F45.9

F34

F10.2

F14.2

F12.2

F15.2

F10.1

F12.1

F14.1

F15.1

F98.5

F50

F95.9

F95.2

F51.0

F51.1

F51.3

F51.9

F51.5

F51.8

F50.9

F50.2

F98.2

F80.9

F43.1

F32.9

F63.9

F63.0

F63.1

F63.2

F63.3

F91.9

F90

F90.9

F81.9

F82

G47.4

G47

F05.9 


\section{FAXBACK RESPONSE}

Name:

\section{Address:}

\section{E-mail:}

Specialty:
FAX: 212.328.0600

MAIL:

CNS SPECTRUMS

MedWorks Media

333 Hudson Street, 7th Floor

New York, NY 10013

\section{Signature:}

Date:

Your comments are important to us. This easy-to-use form provides you with the opportunity to express your opinions. Our goal is to make CNS Spectrums your source for practical and clinical neuropsychiatric information. By filling out this FaxBack form, you will enable us to incorporate your views about our editorial content in future issues. Please fill out this form in its entirety. Thank you.

1. On a scale of 1 to 5 (1=Poor, $5=$ =xcellent), please indicate your level of interest and/or satisfaction with the editorial content in this issue.

\section{Cover Story}

$\begin{array}{lllll}1 & 2 & 3 & 4 & 5\end{array}$

\section{Departments}

CNS News

$\begin{array}{lllll}1 & 2 & 3 & 4 & 5\end{array}$

CME

$\begin{array}{lllll}1 & 2 & 3 & 4 & 5\end{array}$

2. Which areas of neuropsychiatry would you like us to cover in the future?
3. Please describe your reading pattern for this issue:

O cover to cover

O skim Table of Contents

$O$ select items of interest

O skim text

$O$ did not read

4. On a scale of 1 to 5 ( $1=$ Incomplete, $5=$ Comprehensive), how would you describe the depth of coverage for this issue?

$$
\begin{array}{lllll}
1 & 2 & 3 & 4 & 5
\end{array}
$$

\section{Any other comments?}

\section{Please indicate your title: \\ psychiatrist \\ neurologist}

When you send us this form, you'll receive one complimentary slide library and one complimentary reference material. Please make your selection below.

\section{SLIDE LIBRARY}

$\square$ Current Uses of Dopamine Agomists

Monotherapeutic Uses for Dopamine Agomists

$\square$ Diagnosis and Treatment of Premenstrual Dysphoric Disorder

$\square$ Managing Psychiatric Illness in the Elderly

$\square$ Diagnosis and Treatment of Anxiety Disorders in Children

$\square$ Optimal Uses of Antidepressants

$\square$ New Developments in the Treatment of Epilepsy

I Immunogenicity of Botulinum Toxin Therapyy

\section{Reference Materials}

$\square$ The Use of Anticonoulsants in the Treatment of Neuropathic Pain

$\square$ Overview of Social Anxiety Disorder (Social Phobia): Recognition and Treatment

$\square$ Advances in Diagnosis and Treatment of PTSD

$\square$ Current Treatments of ADHD

$\square$ Current and Emerging Treatments for Cervical Dystonia

The Black Book of Psychotropic Dosing and Monitoring 2000 